\title{
Exploring QU Health Students' Experiences of Burnout, Anxiety and Empathy during the COVID-19 Pandemic: A Mixed Method Study
}

\section{Ruba Sulaiman', Sara Ismail1', Maguy ElHajj', Mujahed Shraim², Tanya Kane ${ }^{3}$, Alla El-Awaisi ${ }^{\text {* }}$}

${ }^{1}$ College of Pharmacy (CPH), Qatar University, Doha, Qatar; *Principal Investigator

${ }^{2}$ College of Health Sciences (CHS), Qatar University, Doha, Qatar, ${ }^{3}$ College of Medicine (CMED), Qatar University, Doha, Qatar

\section{Begciggroustel}

- Student burnout is defined as "feeling exhausted because of study demands, having a cynical and detached attitude toward one's study, and feeling incompetent as a student"

- Burnout is a growing concern amongst healthcare students, and it has many impacts on their mental health due to the high demands of the medical and health education

- Multiple studies reported positive association between anxiety symptoms and emotional exhaustion and cynicism subscales

- The use of coping strategies decreases the burnout and promotes resilience

- Due to the COVID-19 outbreak, Qatar University halted all classes and shifted to online delivery which may have contributed to increasing the risk of burnout and declining of academic performance

\section{Objectives}

To assess and describe burnout amongst QU Health students during the COVID-19 pandemic

2) To assess the relationship between burnout and anxiety with empathy amongst QU Health students

3) To determine the coping strategies of QU Health students during COVID-19 pandemic

\section{Method}

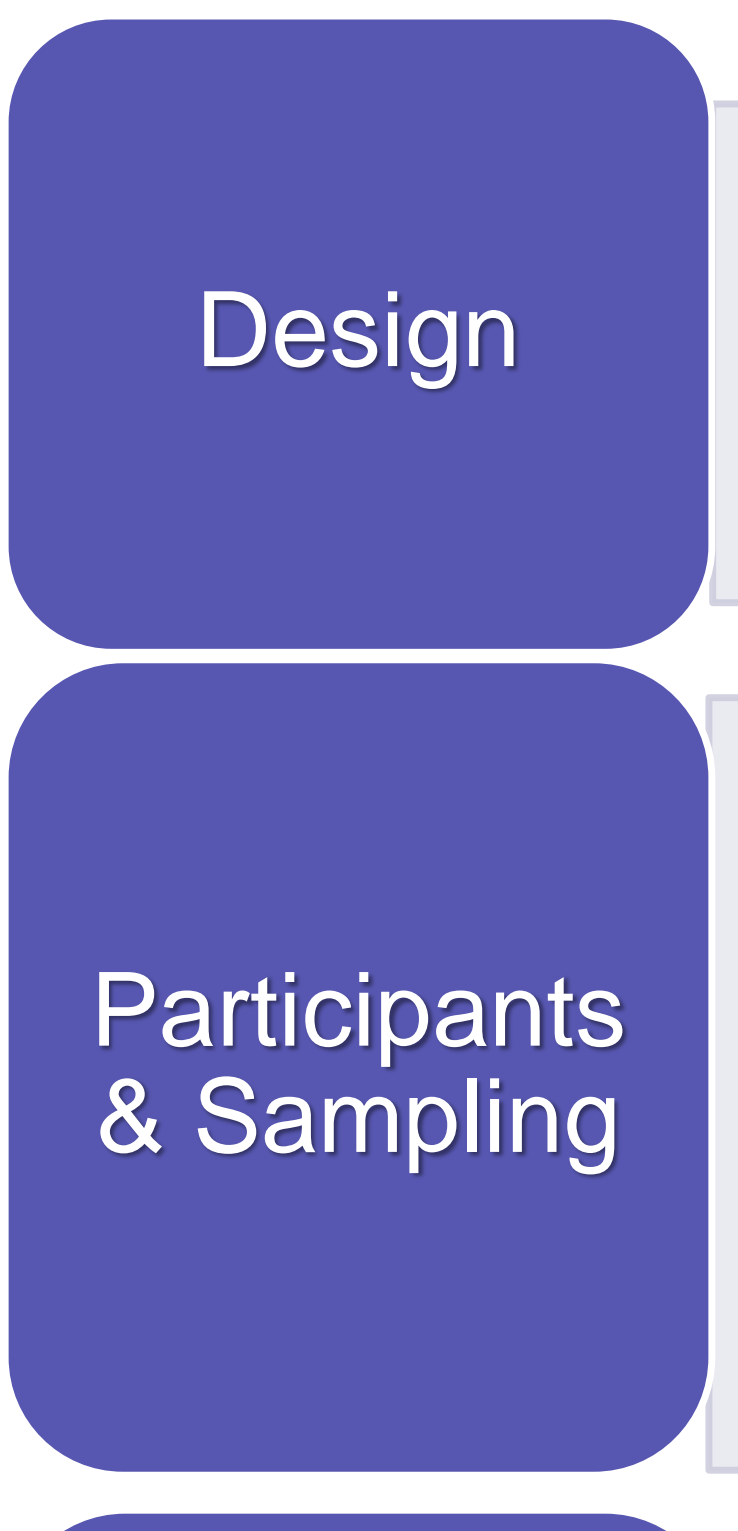

Convergent mixed-methods study

Inclusion: QU Health students ( $\mathrm{n}=1268)$ Exclusion: Students enrolled in fall 2020 Sampling: Population sampling

Settings: Qatar University Self-administered online survey that included 6 sections, including: Maslach Burnout Inventory-General Students Survey (MBI-GS(S)), Interpersonal Reactivity Index (IRI),

Setting and Generalized Anxiety Disorders 7-item scale (GAD-7)

Focus groups were conducted virtually, audio-recorded and transcribed and used the coping reserve model as a framework for data collection and analysis

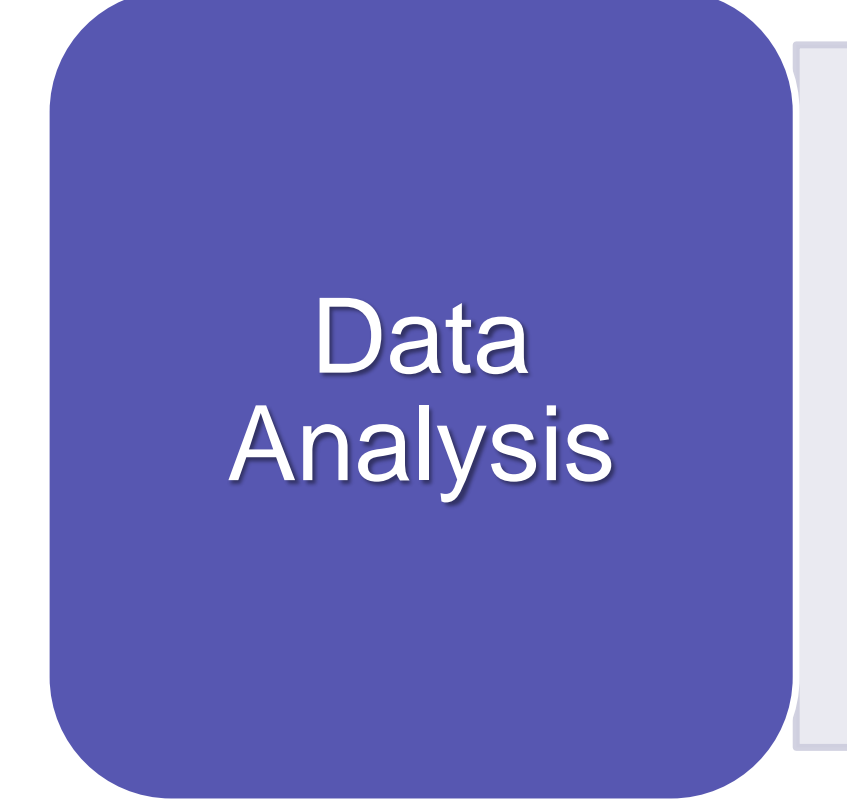

Quantitative Data: Descriptive statistics and multivariate regression analysis Qualitative Data: Deductive thematic analysis

Qatar University Institutional Review Board (QU-IRB)

\section{Resultis}

272 recorded responses for the survey which accounts for $21.45 \%$ response rate and participants characteristics are shown in table 1

- MBI subscales were assessed individually against GAD-7 statuses (as shown in table 2) and against IRI for association with each

Figures 1 and 2 represent responses from the students in regards their courses/semester status and their concern about COVID-19 infection, respectively

8 focus groups were conducted with 3-10 students each thematic analysis was mapped out based on the coping reserve model as shown in Figure 3

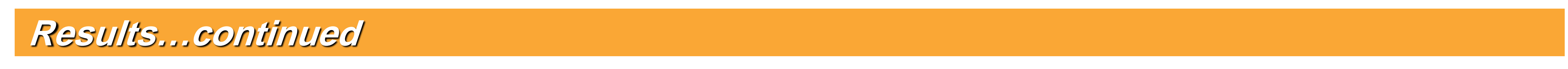

\section{- Table 1. Participant characteristics}

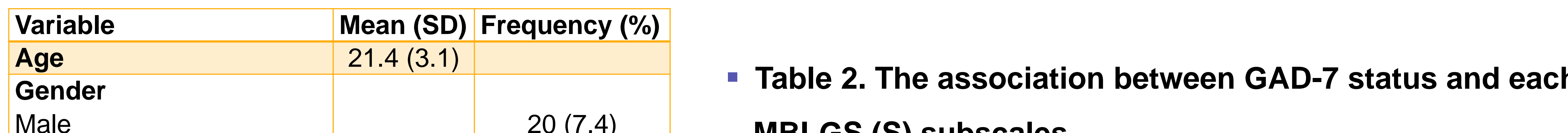

\begin{tabular}{|l|r|r} 
Gender & $20(7.4)$ & MBI-GS (S) subscales \\
\hline Male &
\end{tabular}

Female

Nationality

Qatari

Program

Biomedical

Dental medicine

General health sciences

$252(92.6)$

$194(71.3)$

Medicine

Nutrition

Pharmacy

Physical therapy

Public health

Level

Undergraduate

Year 1

Year 1

Year 3

Year 3

Year 4
Year 5

Year 5
Year 6

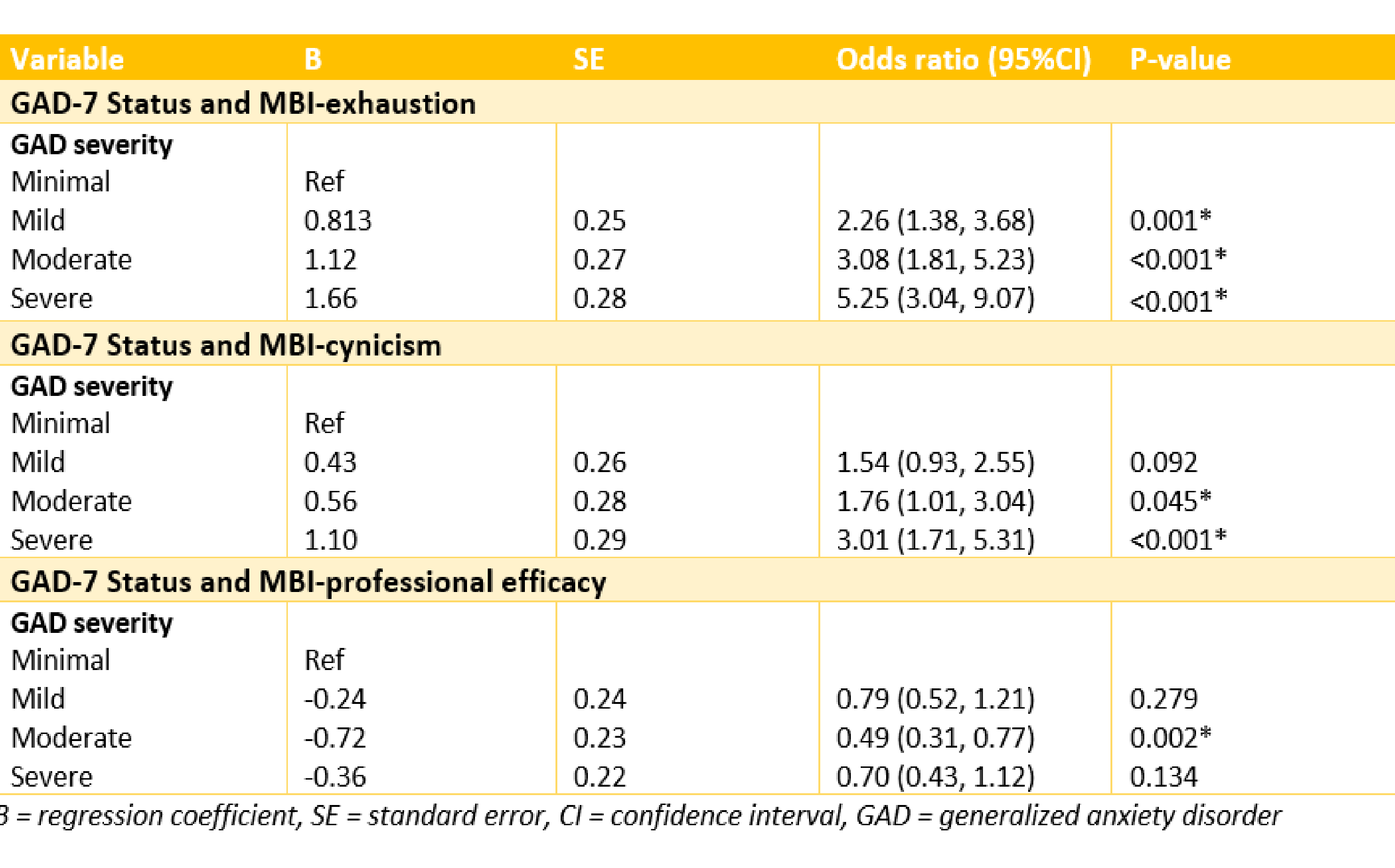

$27(9.9)$

$12(4.4)$

$16(5.9)$

$67(24.6)$

$23(8.5)$

$99(36.4)$

$8(2.9)$
$20(7.4)$

$(88.6)$

$31(88.6)$

$95(34.9)$

$95(34.9)$

$43(23.2)$

$33(12.1)$

$33(12.1)$
$22(8.1)$

$11(4.0)$

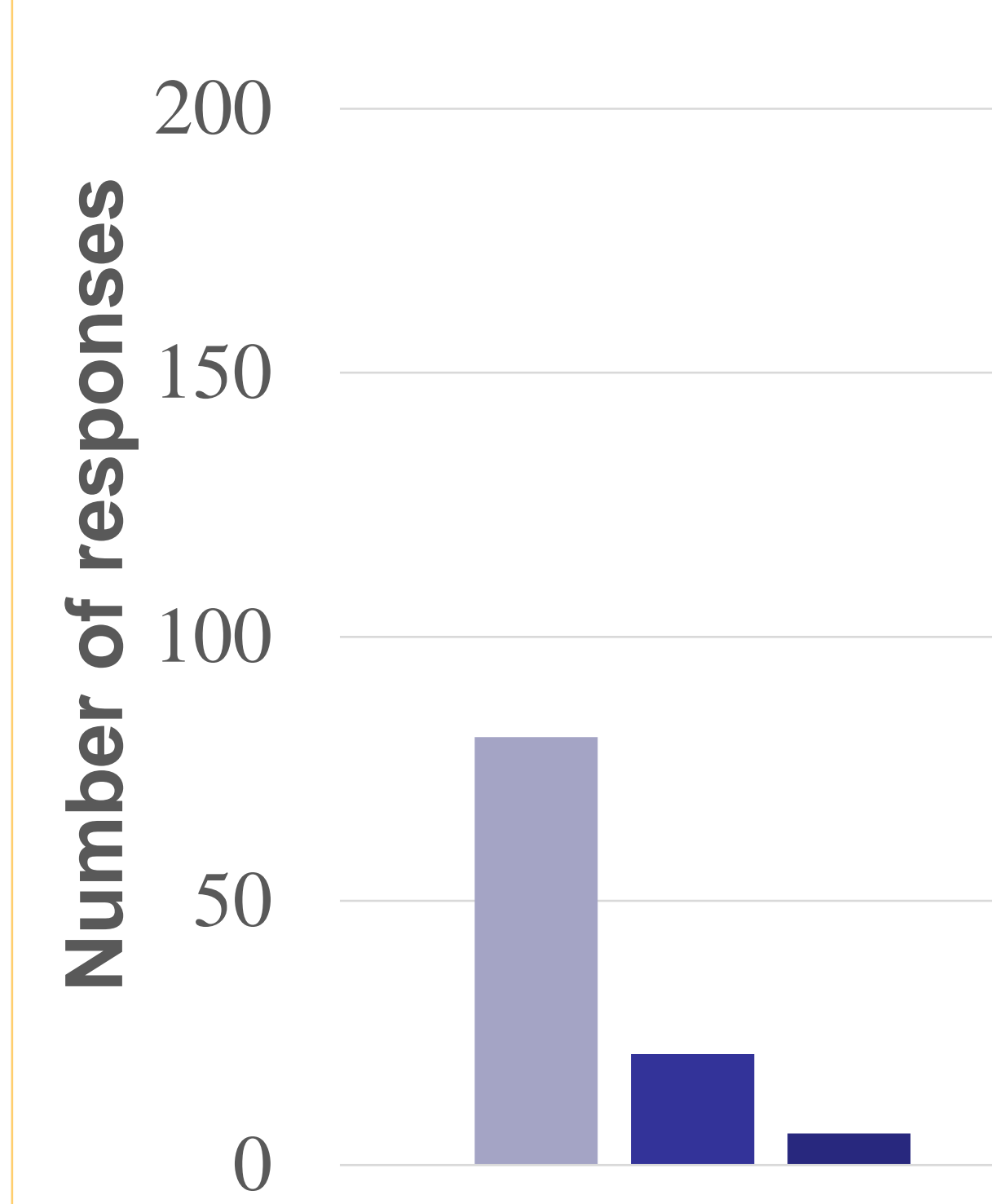

yes

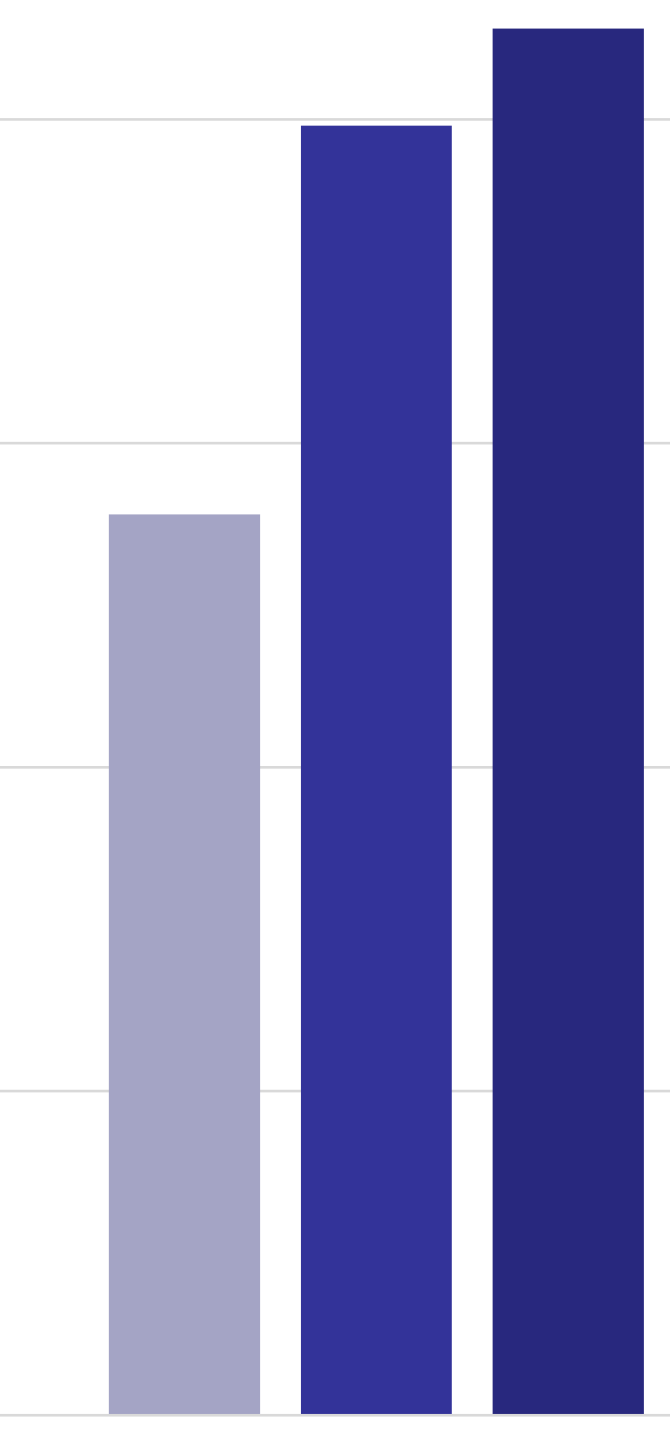

no

withdrawing thoughts

- withdrawn from courses

- withdrawn from semeste

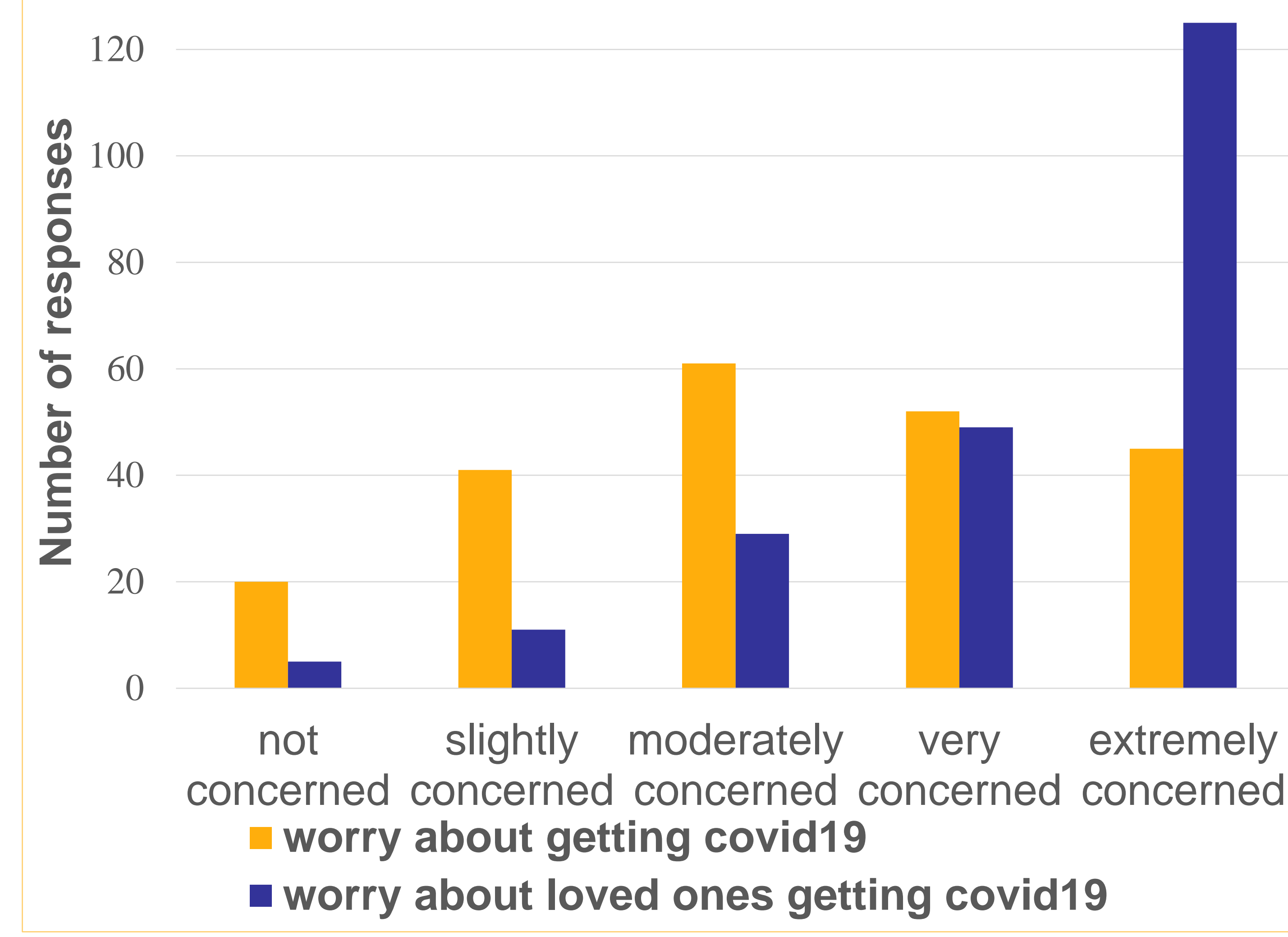

Figure 2. Concerns of COVID-19 infection
Negative input

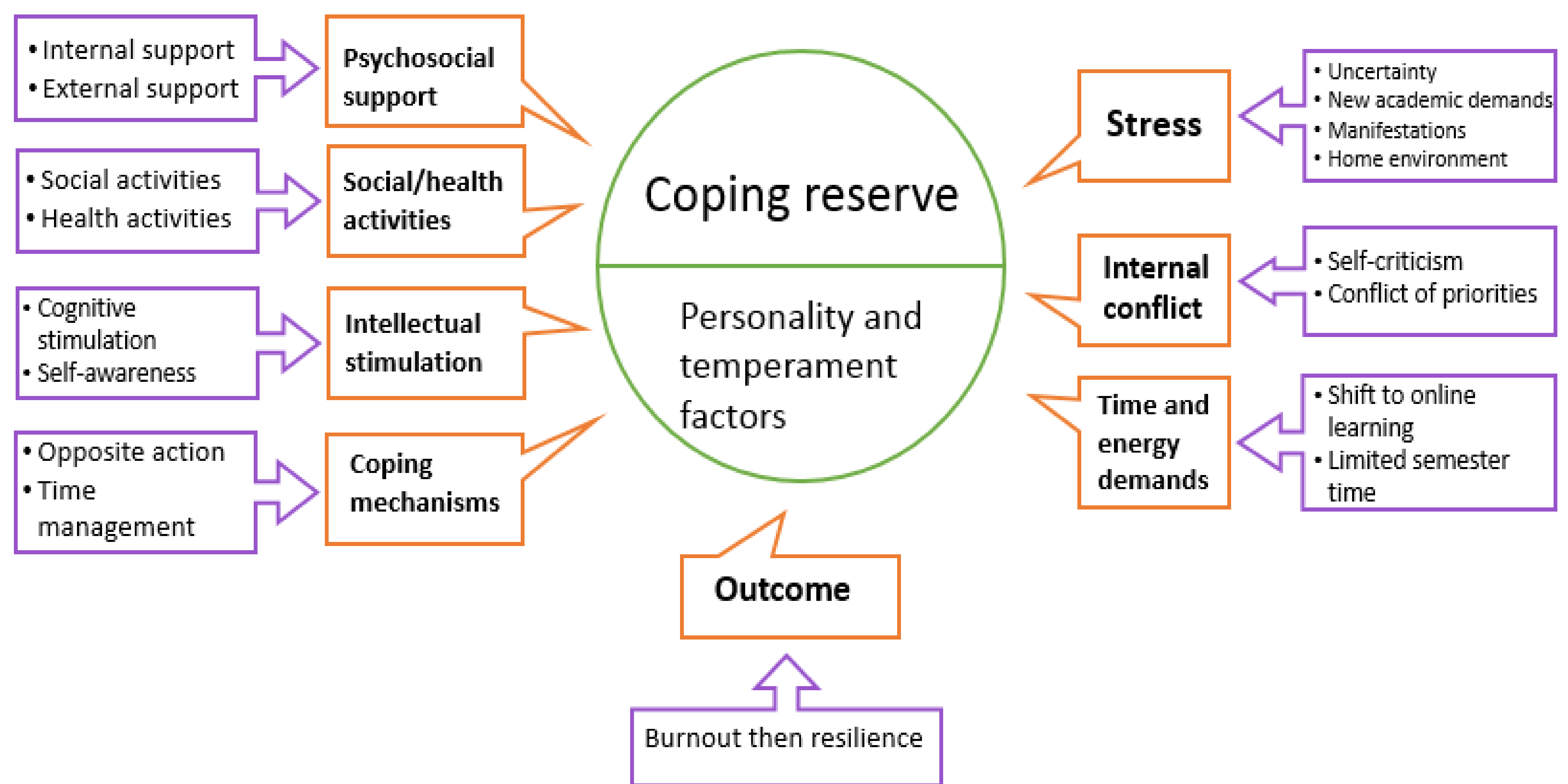

Figure 3. Thematic analysis using the coping reserve model

Constusjons

levels. Additionally, Burnout was associated with some of the IRI subscales

- Most prominent themes extracted from focus group discussions were uncertainty, manifestations of stress, and the shift to online learning

- Students used different coping strategies to adapt with the pandemic and build resilience

- Recommended actions: mentorship program, provision of training on online learning delivery

- Limitations: Small response rate and the survey length

- Recommendation for future research: future research investigating burnout on non-healthcare students,

university faculty members, and investigating the effect of students' burnout on professionalism

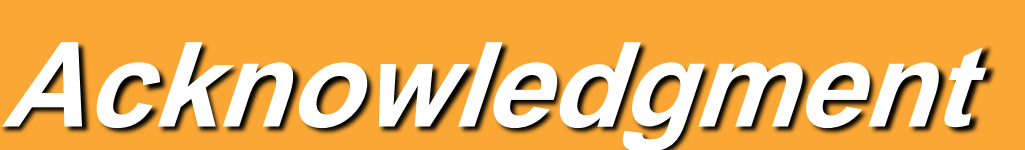

We would like to thank all students who have participated in this study and all the faculty who provided their constructive feedback on the survey: Ms. Myriam Eljaam, Ms. Layan Sukik, Dr. Monica Zolezzi, Ms. Reem EL Hage, Dr. lily O'Hara, and Ms. Sawsan 